\title{
Enseñanza del Algebra Lineal en carreras de ingeniería: un análisis del proceso de la modelización matemática en el marco de la Teoría Antropológica de lo Didáctico.
}

\author{
Teaching Linear Algebra in Engineering Careers: an analysis of the process of \\ mathematical modeling within the framework of the Anthropological Theory of \\ Didactics
}

Fermín Álvarez-Macea ${ }^{\mathrm{a}}$, Viviana Angélica Costa ${ }^{\mathrm{b}}$

\begin{abstract}
${ }^{a *}$ Candidato a doctor en ciencias de la educación, Universidad Nacional de La Plata, Argentina, Docente, Universidad de Antioquia. 0000-0002-2451-9144, fermin.alvarez@udea.edu.co, Antioquia, Colombia

bDra en Enseñanza de las ciencias, Universidad Nacional de La Plata, La Plata, Argentina. 0000-0003-1782-5378, vacosta@ing.unlp. edu.ar, La Plata, Argentina
\end{abstract}

Forma de citar: Álvarez-Macea, F. Costa, V.A. Enseñanza del Algebra Lineal en carreras de ingeniería: un análisis del proceso de la modelización matemática en el marco de la Teoría Antropológica de lo

Didáctico. Eco Matemático, 10 (2), 65-78

Recibido: 5 de marzo de 2019

Aceptado: 10 de mayo de 2019

\section{Palabras clave}

Didáctica, Álgebra

Lineal, Ingeniería, Modelización

Matemática, Metodología de enseñanza.
Resumen:El artículo se enmarca dentro del proceso de modelización matemática como un tema de investigación emergente en didáctica de las matemáticas, a partir de la enseñanza del Álgebra Lineal, en el marco de la Teoría Antropológica de lo Didáctico (TAD). Las Actividades de Estudio y de Investigación (AEI), como dispositivos didácticos dentro de la TAD, propone estudiar las matemáticas a partir de un proceso que se organiza para dar respuestas a las preguntas que surgen de los problemas y así construir los Objetos Matemáticos (OM). Para lograrlo se contempla un conjunto de praxeologías, teorías, tecnologías y tareas. En este orden, la modelización matemática en el marco de la TAD, es un proceso, que va a surgir, emerger, naturalmente cuando se implementan estos dispositivos didácticos, como una nueva alternativa de enseñanza en el modelo de la pedagogía de la investigación y del cuestionamiento del mundo.

*Autor para correspondencia: fermin.alvarez@udea.edu.co 


\section{Keywords}

Didactics, Linear

Algebra, Engineering, Mathematical Modeling, Teaching methodology.

\begin{abstract}
The article is framed within the mathematical modeling process as an emerging research topic in mathematics didactics, based on the teaching of Linear Algebra, within the framework of the Anthropological Theory of Didactics (TAD). The Study and Research Activities (AEI), as didactic devices within the TAD, propuso to study mathematics from a process that is organized to give answers to the questions that arise from the problems and thus build the Mathematical Objects (OM) To achieve this, a set of praxeologies, theories, technologies and tasks is contemplated. In this order, the mathematical modeling in the framework of the TAD, is a process that will emerge, emergent, naturally when these didactic devices are implemented, as a new teaching alternative in the pedagogy model of research and questioning the world.
\end{abstract}

\section{Introducción}

La sociedad está continuamente sometida a diversos cambios de tipo científico tecnológico, social y económico, y la educación no es ajena a ellos. Diferentes investigaciones revelan la importancia de la búsqueda de propuestas alternativas y teorías que sustenten un cambio en lo didáctico en la enseñanza en el ámbito escolar.

La asignatura Álgebra Lineal es fundamental en los estudios de la Ingeniería aplicada. Una diversidad de situaciones problemas y aplicaciones de Ingeniería pueden ser abordados desde los ejes temáticos de vectores, matrices y sistemas lineales de ecuaciones, espacios vectoriales, y transformaciones lineales. Sus orígenes se remontan a las civilizaciones antiguas egipcia, babilónica y china (Luzardo \& Peña, 2006) y desde entonces se ha desarrollado de forma exponencial hasta nuestros días (Tenorio et al., 2011). El potencial del álgebra lineal para entender, explicar conceptos fundamentales y facilitar los cálculos en diferentes ingenierías y varios campos como la computación, la economía, la estadística, la matemática y la física (Lay, 2007). Por consiguiente, el Álgebra Lineal es una de las asignaturas con mayor capacidad de aplicación, se constituye en una herramienta esencial para un ingeniero en cualquier campo disciplinar (Rojas \& Cano, 2009; Costa et al., 2018; Liern, 2018).

Por lo que, a nivel educativo, el Álgebra Lineal posibilita al estudiante una gran alternativa para resolver problemas de la vida cotidiana y algunas ciencias, de ahí que su enseñanza es de vital importancia en los currículos de los programas de ingeniería. Su estudio específico se localiza en diferentes áreas del núcleo de formación del ingeniero, como, por ejemplo: Análisis estructural, termodinámica, programación lineal, control, mecánica de fluidos, entre otras (ACOFI, 2004).

En sintonía con lo anterior, la asignatura Álgebra Lineal en lo que concierne a su enseñanza se caracteriza por ser tediosa y difícil sin importar cual sea la orientación que se dé a la asignatura (matricial, axiomática, geométrica, computacional) esto corresponde a las falencias conceptuales y al tipo de pensamiento requerido para su comprensión (Costa \& Vacchino, 2007; Mola et al., 2013). El componente epistemológico del Álgebra Lineal, las dificultades respecto a los esquemas didácticos y la utilización de varios lenguajes son ejemplos de inconvenientes que se presentan (Oktaç \& Trigueros, 2010). 
Se estudia el Algebra Lineal en diversos programas académicos de ingeniería, en donde sus microcurrículos carecen del proceso de la modelización, dejando solo la parte operativa y abstracta de los contenidos de esta asignatura. Es decir, existe un distanciamiento entre las destrezas o competencias matemáticas que demanda el ingeniero, especialmente la modelar, y las que se presentan en los cursos de Matemática, donde su mayor énfasis son los procesos mecánicos y algorítmicos (Brito-Vallina et al., 2011).

En correspondencia con lo anterior, y teniendo presente las directrices impartidas por el Ministerio de Educación Nacional (MinEducación), en relación con los planes de estudios para la ingeniería plantea que: las ciencias básicas, establecen los cursos de ciencias y matemáticas donde se fundamentan los cimientos y compendios teóricos de la ingeniería, contienen materias que estudian las particularidades y aplicaciones de las ciencias básicas para asentar el diseño de sistemas y mecanismos en la solución de situaciones problemas (Decreto 792, 2001).

Teniendo presente las finalidades de los programas de ingeniería, el proceso de modelización en la enseñanza del Algebra Lineal es un pilar importante, en el contexto de la educación colombiana, tal como lo evidencia (Uzuriaga \& Martínez, 2016) al plantear que en un curso de Álgebra Lineal se inicia por el tema de sistemas de ecuaciones lineales, centrando sus evaluaciones para comprobar si los estudiantes han aprendido los algoritmos para resolver estos sistemas, dejando de lado las aplicaciones que tienen en la solución y modelación de problemas en diferentes áreas del conocimiento o situaciones cotidianas, impidiendo el desarrollo de su pensamiento, de transitar de lo concreto a lo abstracto, sin la posibilidad para argumentar, explicar, construir o demostrar.

En sintonía con lo anterior, Rendón (2016) menciona la necesidad de articular la formación en ingeniería y la formación matemática en su preocupación por fortalecer la formación de ingenieros en toda actividad ingenieril, donde el proceso de modelización sea un medio para cubrir las demandas del ámbito disciplinar y determinar disposiciones a partir de la solución de problemas de una situación del mundo real.

El interés de impartir un conocimiento de manera adecuada y hacer menos abstracta la adquisición del conocimiento en temáticas que conforman los microcurrículos de las asignaturas de las ciencias básicas, tales como cálculo diferencial, integral, vectorial, ecuaciones diferenciales, álgebra y programación lineal, entre otras asignaturas, siempre ha sido una preocupación de la formación del profesional en educación superior. Por lo que las matemáticas deben enseñarse indispensable apelando a situaciones de la vida real tanto físicas como sociales (Ruiz, 2001).

Adicionalmente, las experiencias con la mediación de las diferentes herramientas TIC, han permitido interactuar con los estudiantes y obtener resultados positivos que se evidencian en la comprensión, visualización y reconstrucción de diferentes contenidos ayudados por la modelización como proceso, que permiten aplicarlos a otros tópicos de sus carreras específicas (Vasco, 2006).

Diferentes estudios han demostrado la importancia y relevancia en la enseñanza de las matemáticas acerca de presentar los conceptos de manera contextualizada, argumentándose que estos se aprenden de una forma significativa $y$ los estudiantes demuestran interés, adquiriendo destrezas en la resolución de situaciones problemas concernientes con su entorno, que en actividades ajustadas solo en matemáticas (Trigueros, 2009), es así, donde a nivel universitario falta la articulación de los contenidos matemáticos con las temáticas específicas de las diferentes ingenierías, por lo tanto un acercamiento por medio de la modelización matemática es un proceso fundamental en alcanzar dicho objetivo. 
Dado lo anterior, se han planteado para un progreso en el aprendizaje, desvincularse de la abstracción y acercarse más a lo concreto, se han puesto en curso e implementado varias estrategias didácticas, metodológicas (Costa \& Vacchino, 2007; Costa y Justo, 2015; Costa, \& Rossignoli, 2017). También desde lo epistemológico en didáctica con la teoría APOE (Oktaç \& Trigueros, 2010; RoaFuentes \& Oktaç, 2010; Parraguez, 2013; Salgado \& Trigueros, 2014; Trigueros et al., 2015) y la Teoría Antropológica de lo Didáctico (en adelante TAD) como es el tema central del manuscrito.

La enseñanza del Algebra Lineal se puede repensar en términos didácticos en los que, la modelización matemática, desde un abordaje de la TAD (Chevallard, 1999), puede ser una alternativa que contribuya a mejorar los procesos formativos en carreras de ingeniería. Según Otero et al. (2013), los interrogantes son esenciales en el asunto de construcción de conocimiento, pero en la enseñanza escolar (Universitaria en este caso) los interrogantes han sido remplazadas por las respuestas. Esta situación de enseñanza dedicada al estudio de respuestas en vez de interrogantes transporta al fenómeno didáctico que Chevallard $(2004,2007)$ ha definido metafóricamente como monumentalización del saber. Esta cuestión consiste en enseñar obras matemáticas como objetos ya creados, transparentes e indiscutibles, obras a las que necesariamente por su carácter monumental, a lo sumo se puede visitar (Otero et al., 2013).

En este orden de ideas, se debe reflexionar sobre la situación emergente entre las diferencias de una enseñanza de las matemáticas escolares y una que este dirigida hacia lo funcional. Por lo tanto, se debe conocer el manejo de instrumentos matemáticos en la modelización en ambiente escolar (desde el lugar de la universidad), entendida como: la elección del lenguaje de los instrumentos o herramientas sobre el lenguaje de los objetos y además la modelización desde una perspectiva de prácticas sociales en lo escolar, la modelización solo es estimada como una actividad que le da un sentido de aplicación a los conocimientos adquiridos en los distintos cursos de matemáticas. En este uso del proceso la modelización el énfasis está en la "aplicaciones reales", es decir, en acercar la "realidad" a la matemática (Suárez \& Cordero, 2010).

Lo anterior permite resignificar contenidos y organizaciones matemáticas $(\mathrm{OM})$ de Algebra Lineal y además el proceso de modelización arrojará características que enlacen diseños de situación desde el lugar de la TAD, este marco ofrece un modelo epistemológico que permite el análisis de la actividad humana en su dimensión institucional. Por lo que se debe revisar la literatura sobre los diversos enfoques investigativos acerca de la modelización matemática, la enseñanza del Algebra Lineal y los aportes de la TAD en la educación superior en cursos de ingeniería.

El planteamiento de la modelización matemática en situaciones intra y extra matemáticas según Pochulu, (2018) y Barquero (2009) ), determinan que un problema de investigación en el marco del campo de la Didáctica de las matemáticas bajo la perspectiva de la TAD, es el estudio de la importancia y de la función de la modelización en la enseñanza de la matemática como algo específico de su planteamiento más general, donde la importancia de cimentar modelos epistemológicos de la acción matemática institucional, la cual es, por lo tanto, apreciada como su objeto principal de investigación. Para dar una respuesta consideran dos categorías: la epistemológica y la ecológica. La epistemológica implica especificar a qué se refieren con modelización matemática y cómo se relaciona con la Matemática. La ecológica, se refiere, a dar exactitudes sobre escenarios que deberían tener en cuenta para que el proceso de la modelización matemática pueda desarrollarse en las instituciones del ámbito escolar.

De lo anterior y con miras a determinar que matemáticas en el contexto colombiano necesitan los 
estudiantes en las diferentes ingenierías y de qué forma se puede repensar la enseñanza de las matemáticas, en este caso específico del Algebra Lineal, junto con el proceso de la modelización matemática, es importante resaltar:

En la misma línea de los planteamientos de la TAD, Rodríguez $(2007,2010)$ y Quiroz \& Rodríguez (2015) plantea que el proceso de modelización se fundamenta en:

(1) La caracterización e identificación de praxeologías relacionadas a la modelización matemática desarrolladas en ocho etapas, y

(2) la incorporación del aspecto del dominio pseudoconcreto para la comprensión del propio proceso de modelización, desde el lugar del estudio que examina la presencia de una transposición de los saberes escolares según la Teoría de Transposición Didáctica (Chevallard, 1985) (ver la figura 1).

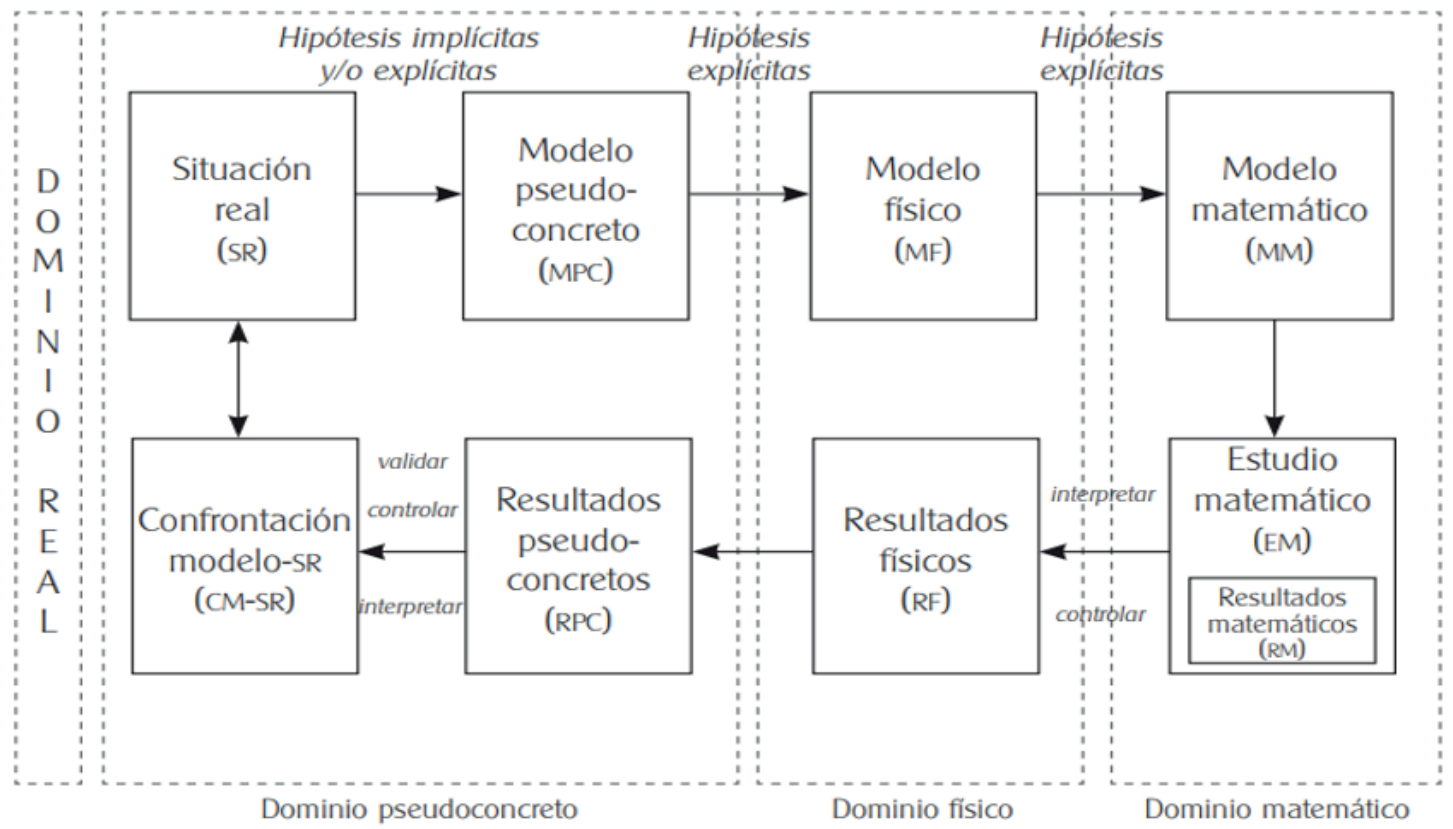

Figura 1. Esquema de modelización Rodríguez (2010) y Quiroz \& Rodríguez (2015)

Teniendo presente los antecedentes investigativos en relación con la enseñanza del Álgebra Lineal, la modelización matemática surgirá como un proceso natural al buscar respuestas a preguntas generatrices relacionadas con problemas actuales de la ingeniería (por ejemplo, situaciones ambientales, de movilidad, de seguridad informática, entre otros).

\section{Estado de la cuestión}

En lo concerniente a la revisión de la literatura sobre modelización matemática y la enseñanza del Algebra Lineal, bajo el marco de la TAD, se tienen investigaciones tales como:

Vázquez et al. (2016), realizan un estudio de las praxeologías, fundamentado en la Teoría Antropológica de lo Didáctico (TAD) de un procedimiento procedente de la ingeniería conocido como Separación Ciega de Fuentes (BSS). En este procedimiento están inmersas praxeologías que pueden incidir en los cursos 
iniciales de matemáticas dentro de la educación formativa de ingenieros, específicamente dentro de una clase de Álgebra Lineal. El estudio determina que la Separación Ciega de Fuentes tiene una latente capacidad para concebir actividades de modelización que relacionen la teoría matemática con el campo de acción ingenieril. Finalmente, se exhibe una idea inicial para una actividad de estudio e investigación(AEI), en el marco de la BSS.

Por su parte, Bosch et al. (2006), introduce determinadas generalidades basadas de la Teoría Antropológica de lo Didáctico, que proveerán herramientas de corte didáctico esenciales para reformar un posible desarrollo del contexto de investigación en lo referente a modelización y aplicaciones matemáticas, además para plantear una reformulación de lo que se concibe como proceso de Modelización matemática.

En relación con la Modelización y la enseñanza del Algebra lineal, Salgado (2015), describe los problemas de abstracción de diferentes temas del Algebra lineal tales como combinación lineal, conjunto y espacio generado, dependencia lineal, base y dimensión. Valores y vectores propios de una matriz, pero a la vez resalta la importancia de estos temas, por sus múltiples aplicaciones dentro y fuera de las matemáticas. Además, presenta una propuesta didáctica para el aprendizaje de dichos conceptos mediante una experiencia en clase apoyada en dos marcos teóricos: Modelos y Modelación y Teoría APOE.

De forma similar, Trigueros (2009), evidencia la relevancia e importancia del tema de la modelización matemática en las aulas de clase y en el contexto universitario. Se despliega algunos puntos de vistas en relación con el papel de la modelización en el salón de clase de matemáticas, además presenta los resultados de ciertas prácticas específicas de su aplicación en la enseñanza a nivel universitario, donde evidencia limitaciones y condiciones existentes de la enseñanza con esta metodología.

Teniendo presente la enseñanza del Algebra Lineal en carreras de ingenierías, Costa \& Rossignoli (2017), establecen la descripción de una perspectiva con que se imparte los conceptos en relación al Álgebra Lineal en una asignatura del Área de Ciencias Básicas en una Facultad de Ingeniería exponiendo las conclusiones de los resultados obtenidos a partir de un cuestionario aplicado a sus alumnos que tiene como objetivo identificar las posibles causas, los posibles inconvenientes en la enseñanza y aprendizaje de dichos conceptos.

En la misma línea, Siero (2017), analiza el rol de la modelización matemática en la educación profesional de ingenieros. Un análisis de las asignaturas de Mecánica Estructural de Materiales Compuestos (MEMC) y de Álgebra Lineal (AL) mostró que dentro del curso de AL hay pocas o nulas tareas de modelación matemática, mientras que en la asignatura de MEMC se proponen tareas que solicitan el uso de herramientas de la asignatura de AL, mostrando una desarticulación entre ambas asignaturas

\section{La modelización matemática y la TAD}

En las trayectorias de enseñanza aprendizaje de la Ingeniería, se emplea a todo momento para hacer representaciones de diversos sistemas reales, la utilización de modelos matemáticos, es decir, se sustituye conceptualmente un método de dominio real formado por objetos materiales por otro formado por objetos de otro dominio, éste formado por sujetos matemáticos (Scardigli et al., 2013).

Villa (2007) determina una discrepancia entre los términos modelización y modelación matemática. Modelización la caracteriza como actividad científica y la modelación la define en términos de una herramienta para construir conceptos matemáticos en el aula de clase. La 
primera (Modelización matemática) ha tenido gran acogida por los matemáticos aplicados como un proceso dinámico que les permite entender diversos problemas o alguna situación de importancia especifica en Física, Química, Biología, entre otras, en cambio que la modelación matemática se concibe como el método en la enseñanzaaprendizaje que utiliza el proceso de modelización en cursos regulares. El término modelación es una "contracción" de los términos modelización y educación, es decir modelación = Modelización + Educación (Bassanezi \& Biembengut, 1997).

En ese marco, se deduce por modelación matemática la actividad que se realiza en la clase de matemáticas cuya naturaleza se emana de la actividad científica de la modelización matemática Villa (2007). Ahora bien, ¿Por qué hacer énfasis en la modelación matemática, teniendo como cimiento la TAD?

Uno de los pilares de la TAD es que toda actividad matemática puede ser explicada como una actividad de modelación (Cumbal, 2012). El modelado de situaciones reales o de contexto involucra a los estudiantes con diferentes procesos sociales, matemáticos y de comunicación, lo que les da una extensa gama de posibilidades de aprendizaje, y por lo tanto los motiva a aprender matemáticas (WunaJeeh et al., 2015).

En este orden de ideas, diversos estudios entre ellos, Bassanezi \& Salett (1997), plantean la importancia y revelan la existencia de la relación entre los conceptos de formalización y modelización matemática. Este primer concepto (formalización) en un problema (real o de contexto) en palabras matemáticas, es casi siempre el momento más difícil de la modelización matemática y debe ser asimilado con la propia práctica; es así, donde no se puede hablar de modelización sin entender cómo es el proceso de la formalización y los aspectos cognitivos que se involucran a la hora de determinar el análisis de una situación que se pretende matematizar con un norte muy bien definido, así mismo, permite desarrollar actividades donde la metacognición, coadyuve al aprendizaje espontaneo por parte de los estudiantes.

De esta manera, es transcendental destacar que, en la búsqueda de la aplicación de la modelización matemática, conlleva a los docentes y estudiantes, a cambiar paradigmas para responder a nuevos desafíos, necesidades, desarrollando en ellos diversas competencias matemáticas, procesos comunicativos y habilidades sociales (Blum \& Borromeo, 2009).

Desde otros puntos de vistas, es importante precisar cómo se asume y definen el concepto de modelización matemática desde perspectivas propias de lo interdisciplinar de las matemáticas hasta el aspecto socio-crítico y cultural:

(1) El proceso de puntualizar en palabras matemáticas una situación del mundo real obteniendo resultados matemáticos y la valoración e interpretación matemática de dicha situación (Gómez-Chacón \& Maestre, 2008).

(2) La modelización es un mecanismo que da sentido a los conocimientos matemáticos mediante sus aplicaciones (Salett \& Hein, 2004).

(3) La modelización matemática como una estructuración teórica que un sujeto realiza al enfrentar una tarea matemática en la que se pone en juego sus conocimientos y como características propias de esta construcción, la modelización posee su propia estructura (Guerrero-Ortiz \& Mena-Lorca, 2015).

(4) La modelización es la clasificación del lenguaje de los instrumentos sobre el lenguaje de los objetos. Desde perspectiva de prácticas sociales en la escuela, es considerada como una actividad que le da un sentido de aplicación a los conocimientos adquiridos en los distintos cursos de matemáticas. 
El énfasis está en las aplicaciones "reales", es decir, en acercar la "realidad" y la "matemática" (Suárez, 2014).

(5) La modelización en el ámbito escolar matemático todavía se propone ésta como un método de enseñanza-aprendizaje en donde los estudiantes pueden desarrollar competencia y capacidades para desenvolverse satisfactoriamente en el mundo actual (Cordero, 2003; Aravena et al., 2008).

(6) Diversos autores académicos establecen a la modelización como un asunto donde los estudiantes aplican matemáticas (Ottesen, 2001), estableciendo que las matemáticas y lo que se define como realidad son dos agregados disjuntos y el modelaje es un medio de conjugarlos, de modo que modelización, modelaje y modelo es considerado un método de investigación y una estrategia de enseñanza-aprendizaje, según (Salett \& Hein, 2004).

En cierta medida parten de la matematización de una temática comprendida como el desarrollo de detallar en términos matemáticos un fenómeno real, adquiriendo resultados matemáticos y la evaluación e interpretación matemática de una situación real (Gómez-Chacón \& Maestre, 2008). (Ver figura 2).

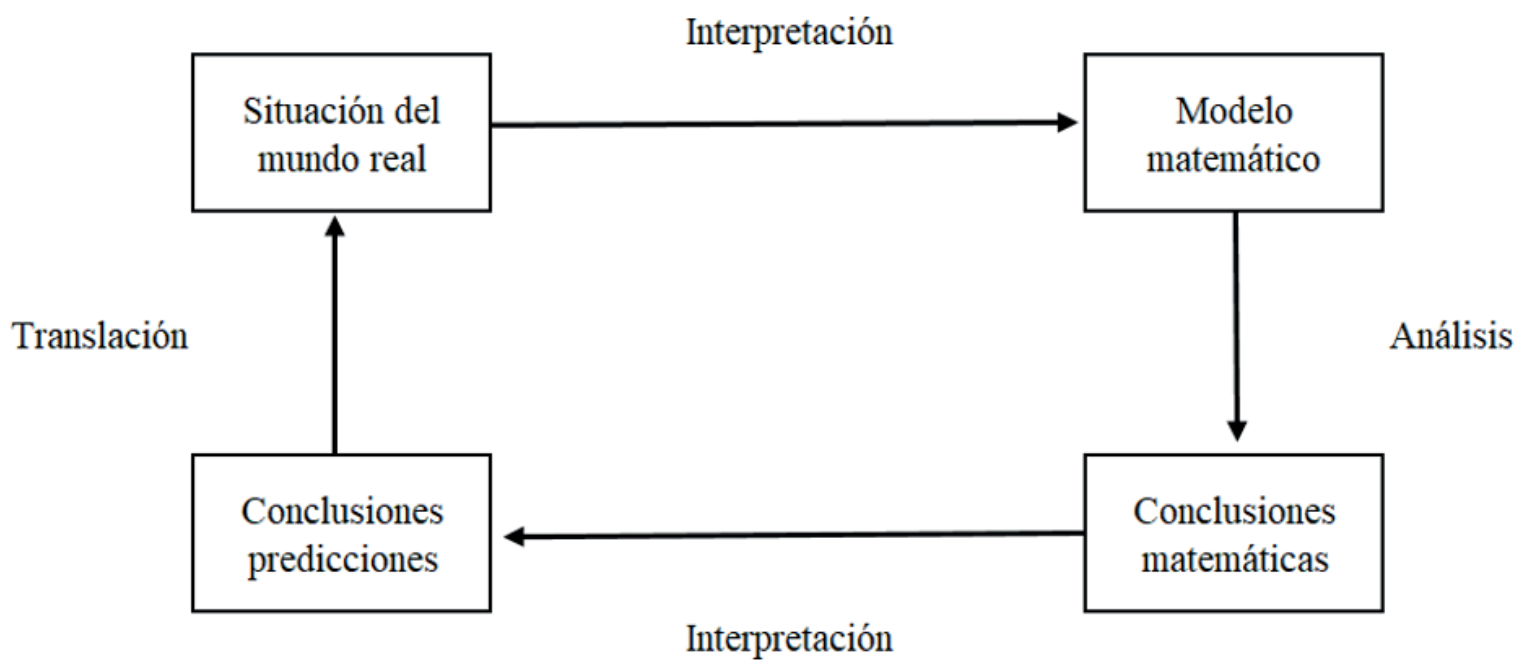

Figura 2. Proceso de Modelización Matemática (Gómez-Chacón \& Maestre, 2008, p.5).

En el marco de la TAD, mediante la concepción de praxeologias todo accionar del ser humano puede ser modelada. Es importante diferenciar dos aspectos inseparables en el concepto de praxeologías: El nivel de la práctica matemática o praxis (saber-hacer) y el discurso lógico sobre la práctica o logos (saber) (Farías, 2015).

Una praxeología se representa mediante cuatro componentes: Clasificación(tipos) de problemas $(\mathrm{T})$, conjunto de técnicas $(\tau)$ o "maneras de hacer", tecnología $(\theta)$, teoría $(\Theta)$. Simbólicamente $P=[T, \tau, \theta, \Theta]$.

Una relevancia de la generalidad de praxeología es que consolida bajo un mismo concepto el "saber" o acumulado organizado de conocimientos y la actividad. Cuando se habla una teoría o disciplina se refiere habitualmente a un acumulado de praxeologías que destinamos metonímicamente a partir de su componente tecnológico-teórico $[\theta, \Theta]$. Al contrario, al referirnos a la "práctica pedagógica", de la "pericia" o del 
"saber hacer" del docente, es resaltar el componente práctico-técnico $[\mathrm{T}, \tau]$ de la praxeología, excluyendo o dejando implícito su elemento tecnológico-teórico (a menudo muy habituado y difícil de describir) (Bosch \& Gascón, 2009).

Según Farías, (2015), el modelo "hacer matemática" radica en impulsar una praxeología matemática, es decir en resolver determinados problemas con diversos tipos de técnicas ("el saber hacer") de carácter comprensible, justificada y razonada (mediante el adecuado "saber").

Entonces de acuerdo con Bosch et al. (2006), todo asunto de estudio, en relación con la actividad humana, puede ser modelado mediante una praxeología designada praxeología didáctica, conformada por varias tareas didácticas problematizadoras, técnicas didácticas para afrontarlas y tecnologías y teorías didácticas que coadyuven a su explicación y respectiva justificación.

\section{Materiales y métodos}

Para alcanzar los objetivos y dar respuesta a la pregunta de investigación, en relación con el enfoque investigativo, el marco teórico y el estado del arte, se propone definir diferentes etapas, herramientas, actividades y técnicas que sean coherentes con la propuesta planteada. En este sentido, se desarrollará una investigación cualitativa, de corte descriptivo e interpretativo, con elementos etnográficos, debido a la importancia de los aspectos sociales y además el contexto en el cual ocurren los acontecimientos (Hernández et al., 2014) considerados de suma importancia dentro de la TAD (Chevallard, 2009).

La investigación apoyada en primera instancia en la epistemología y recorrido bibliográfico acerca del proceso de Modelización matemática en la enseñanza del Algebra Lineal, mediados por recursos de las nuevas tecnologías de la información y la comunicación. En segunda instancia definir y diseñar unas AEI en la búsqueda de respuesta al problema, que hagan surgir naturalmente el proceso de modelización matemática, que permita reconstruir OM del Algebra Lineal y en una tercera, registrar y analizar los datos de la información resultante, donde los agentes participes de la investigación, reflexionen ,construyan y reconstruyan en un contexto natural de interacciones con los conocimientos adquiridos, direccionados bajo la luz de los objetivos planteados en esta propuesta de investigación.

Es importante resaltar que, en esta investigación cualitativa, las partes que la componen no son sujetos inertes sino activos dentro de un mundo académico netamente interactivo, donde las observaciones, proposiciones y argumentaciones son un engranaje de la investigación a desarrollar.

Participantes y escenario de la Investigación

En la presente propuesta de investigación, aplicando la técnica de observación participante, los participantes hacen referencia a los estudiantes y el docente-investigador, de los programas de ingeniería de la institución de educación superior, de carácter público (oficial) Politécnico Jaime Isaza Cadavid (PJIC) en la ciudad de Medellín (Colombia), matriculados en dos grupos de la asignatura Algebra Lineal, la cual está ubicada en el tercer y cuarto semestres, según el programa, de los planes de estudio.

Es importante resaltar, que el desarrollo de esta propuesta se llevará a cabo en grupos heterogéneos en lo que concierne a los programas académicos que cursan los estudiantes. Así, en un mismo curso se pueden encontrar estudiantes de ingeniería de Productividad y Calidad, Civil, Higiene y Salud ocupacional, Sistemas, Instrumentación y Control. Además, un pequeño porcentaje de los estudiantes tienen formación previa (técnica o tecnológica) en áreas relacionadas con las ingenierías que cursan actualmente.

La población estudiantil del Politécnico Jaime Isaza Cadavid, tienen en promedio entre 35 y 40 
estudiantes por grupo, con edades entre 18 y 24 años, con un porcentaje de $52.84 \%$ de género masculino y $38.85 \%$ de género femenino, en sede central, poblado (Ver tabla 2), provenientes de colegios públicos (en su mayoría) y privados de la ciudad de Medellín y su área metropolitana, de estrato socioeconómico 1,2 y 3 en su mayoría (Ver figura 3).Específicamente en los cursos de Álgebra lineal, impartidos por el docente investigador, tienes las siguientes características.

Tabla 1. Características de los estudiantes de los cursos de Álgebra Lineal.

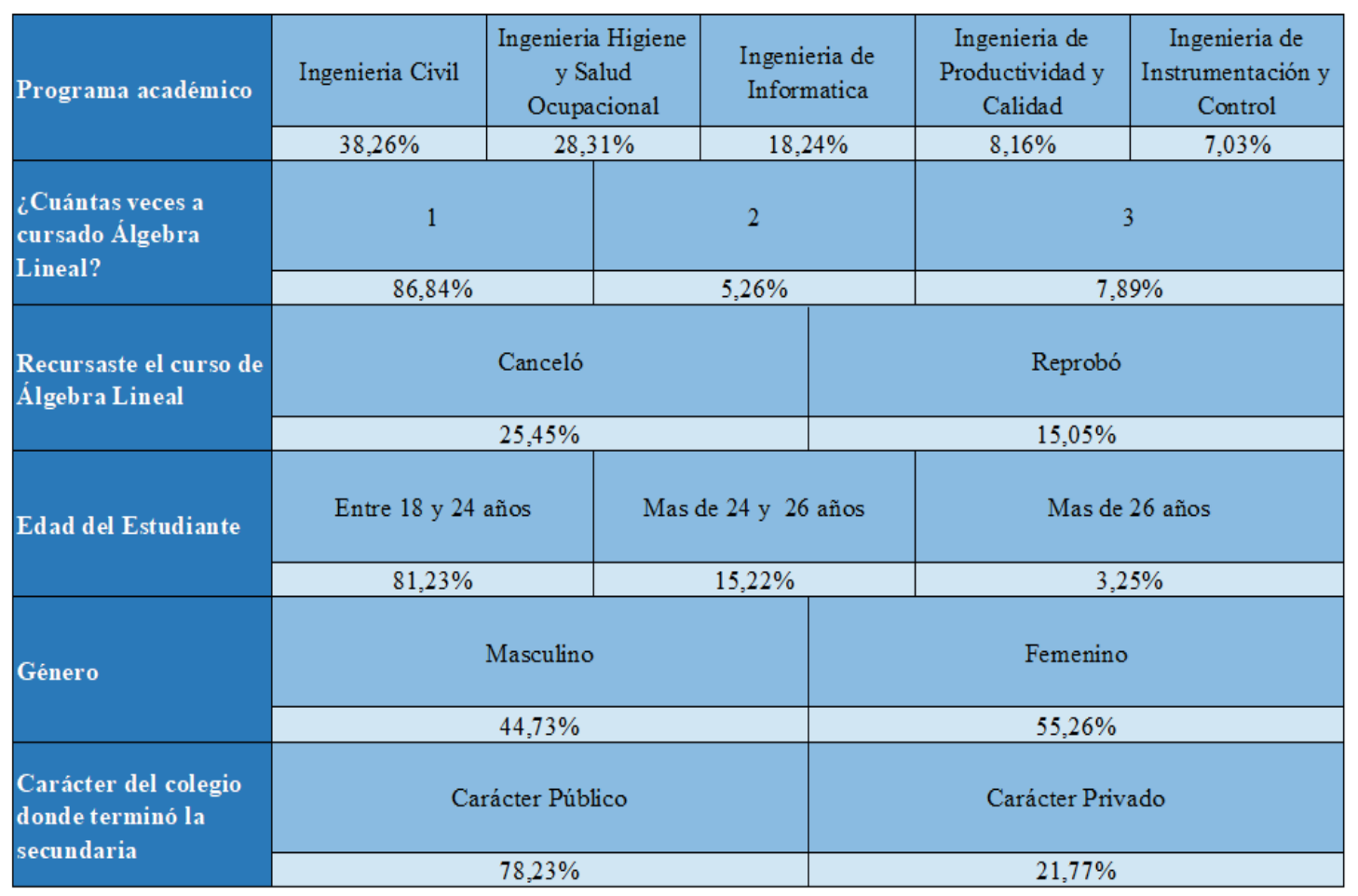

Tabla 2: Estudiantes matriculados clasificación por género Fuente: Sistema Académico Universitas XXI-2019-2

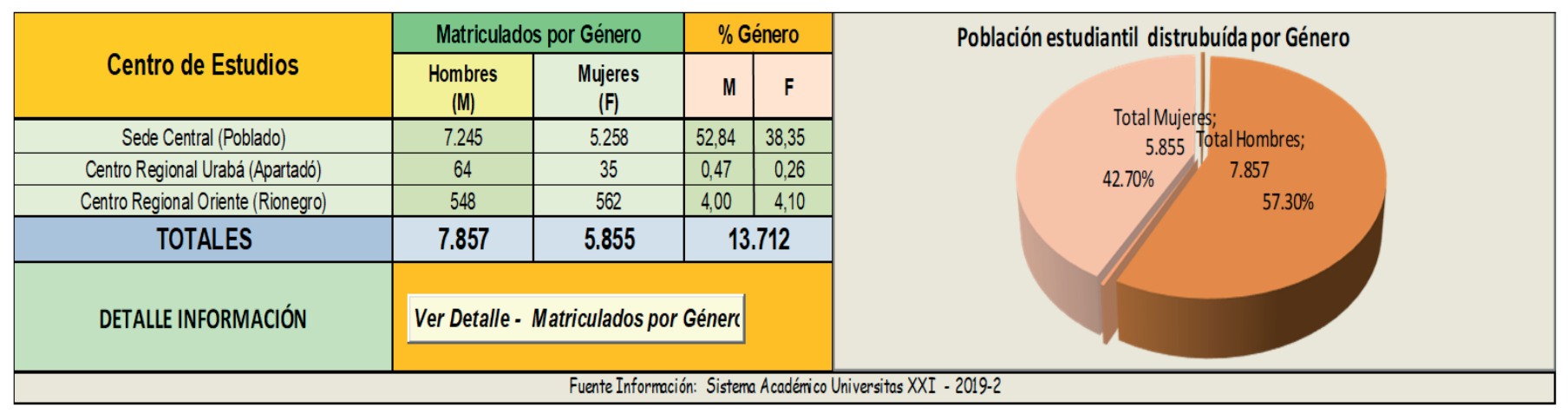


Tabla 3: Estudiantes matriculados clasificados por Estrato socio-económico. Fuente: Sistema Académico Universitas XXI-2019-2

\begin{tabular}{|c|c|c|c|c|c|c|c|c|c|}
\hline \multirow{12}{*}{$\begin{array}{c}\text { MA TRICULADOS } \\
\text { POR } \\
\text { ESTRA TOY } \\
\text { CENTRODE ESTUDIOS } \\
\text { (Pregrado) }\end{array}$} & \multicolumn{9}{|c|}{ Estudiantes Matriculados Distribuidos por Estrato Socioeconomico. Periodo 2019-2 } \\
\hline & \multirow{2}{*}{ Estrato } & \multicolumn{2}{|c|}{ Poblado } & \multicolumn{2}{|c|}{ Apartadó } & \multicolumn{2}{|c|}{ Rionegro } & \multirow{2}{*}{ Total } & \multirow{2}{*}{ Total \% } \\
\hline & & Total & Total \% & Total & Total $\%$ & Total & Total \% & & \\
\hline & 1 & 1.644 & $13 \%$ & 43 & $50 \%$ & 52 & $5 \%$ & 1.739 & $13 \%$ \\
\hline & 2 & 5.823 & $47 \%$ & 39 & $45 \%$ & 468 & $42 \%$ & 6.330 & $46 \%$ \\
\hline & 3 & 3.927 & $31 \%$ & 4 & $5 \%$ & 553 & $49 \%$ & 4.484 & $33 \%$ \\
\hline & 4 & 399 & $3 \%$ & 0 & $0 \%$ & 34 & $3 \%$ & 433 & $3 \%$ \\
\hline & 5 & 104 & $1 \%$ & 0 & $0 \%$ & 0 & $0 \%$ & 104 & $1 \%$ \\
\hline & 6 & 15 & $0 \%$ & 0 & $0 \%$ & 0 & $0 \%$ & 15 & $0 \%$ \\
\hline & Sin estrato & 591 & $5 \%$ & & $0 \%$ & 16 & $1 \%$ & 607 & $4 \%$ \\
\hline & Total & 12.503 & $100 \%$ & 86 & $100 \%$ & 1.123 & $100 \%$ & 13.712 & $100 \%$ \\
\hline & $\begin{array}{r}\text { De acuerdo } \\
\text { Estra }\end{array}$ & $\begin{array}{l}\text { antes matricu } \\
\text { ional Urabá ( }\end{array}$ & $\begin{array}{l}\text { restrato, se } \\
\text { o) el Estrato }\end{array}$ & $\begin{array}{l}\text { que en la } \\
\text { neral, de lc }\end{array}$ & $\begin{array}{l}\text { la (Poblado) } \\
\text { studiantes me }\end{array}$ & $\begin{array}{l}\text { l Estrato } \\
\text { la mayoria }\end{array}$ & $\begin{array}{l}\text { regional Ori } \\
\text { pertenecen a }\end{array}$ & $\begin{array}{l}\text { Rionegro) } \\
\text { ato } 2 \text {. }\end{array}$ & \\
\hline & & & Fuente Inform & tema Acadé & itas XXI - 20 & & & & \\
\hline
\end{tabular}

\section{Resultados}

Con el desarrollo de la investigación en curso se pretende alcanzar resultados acordes a los objetivos e hipótesis planteadas que estén direccionadas en dar respuestas a las preguntas de investigación y por ende permitir futuras investigaciones en la misma línea.

En este orden de ideas, los resultados esperados son:

La construcción de los objetos matemáticos con sentido, en relación con la modelización con temas del Algebra Lineal.

De igual forma, los aspectos relevantes y las potencialidades de las implementaciones de los componentes (constructos) de la Teoría Antropológica de lo Didáctico (TAD) tales como las praxeologías, lo fundamentos didácticos, permitan evidenciar que una enseñanza del algebra lineal por medio del Modelo Praxeológico de Referencia (MPR) y las AEI, son una alternativa que permite el cambio en los roles tanto del estudiante como del docente en su participación activa y de este modo se pase de una educación tradicional y menos abstracta.

Por consiguiente, la implementación de la pedagogía de investigación y cuestionamiento del mundo va permitir que las situaciones problemas no van hacer idealizadas o propuestas de forma cerradas o acabadas, sino que el estudiante identifique, plantee y resuelva problemas del mundo de la ingeniería en términos y condiciones reales.

\section{Conclusiones}

En este trabajo se describe el estado de arte o de cuestión, sobre las temáticas de modelización matemática en el marco de la Teoría Antropológica de lo Didáctico (TAD) y además aspectos metodológicos y didácticos sobre la investigación doctoral en desarrollo concerniente con la enseñanza de Álgebra Lineal, la cual se llevará a cabo en la facultad de ingeniería del Politécnico Jaime Isaza Cadavid (PJIC) en la ciudad de Medellín Colombia.

El dispositivo didáctico contemplado en la TAD como son las Actividades de Estudio e Investigación (AEI), va a permitir que surjan de forma natural los procesos de modelización y su respectivo análisis por medio de praxeologias y dialécticas en temáticas propias del Álgebra Lineal en carreras de Ingeniería.

Con esta investigación se pretende contemplar nuevas alternativas de enseñanza a nivel de la educación superior, contrarrestando la educación tradicional por medio de la incursión de la Pedagogía de Investigación y Cuestionamiento del Mundo (PICM), donde los estudiantes tengan un rol más activo y asignaturas como el Álgebra Lineal, sea 
una gran herramienta significativa en su formación ingenieril.

\section{Referencias}

ACOFI (2004). Contenidos programáticos básicos para ingeniería. Bogotá: Acofi.

Aravena, M., Caamaño, C., \& Giménez, J. (2008). Modelos matemáticos a través de proyectos. Revista latinoamericana de investigación en matemática educativa, 11(1), 49-92.

Barquero, B. (2009). Ecología de la Modelización Matemática en la enseñanza universitaria de las Matemáticas. España: Universitat Autònoma de Barcelona.

Bassanezi , R., \& Salett, M. (1997). Una modelación matemática: Una antigua forma de investigaciónun nuevo método de enseñanza. Revista de didácticas de las matemáticas, (32), 13-25.

Bassanezi,R.,\&Biembengut,M.(1997). Modelación matemática: Una antigua forma de investigación un nuevo método de enseñanza. Números. Revista de didáctica de las matemáticas, 32, 1325.

Blum, W., \& Borromeo, R. (2009). Mathematical Modelling: Can It Be Taught And Learnt? Journal of Mathematical Modelling and Application, 1(1), 45-58.

Bosch, M. \& Gascón, J. (2009). Aportaciones de la Teoría Antropológica de lo Didáctico a la formación del profesorado de matemáticas de secundaria. En M.J. González, M.T. González \& J. Murillo (Eds.) Investigación en educación matemática XIII (pp. 89-114). Sociedad Española de Investigación en Educación Matemática, SEIEM.

Bosch, M., García, F., Gascón, J. \& Ruiz, L. (2006). La modelización matemática y el problema de la articulación de la matemática escolar. Una propuesta desde la teoría antropológica de lo didáctico. Educación matemática, 18(2), 37-74.

Brito-Vallina, M., Alemán-Romero, I., FragaGuerra, E., Para-García, J., \& Arias-de Tapia, R. (2011). Papel de la modelación matemática en la formación de los ingenieros. Ingeniería
Mecánica, 14(2), 129-139.

Chevallard, Y. (1985). La transposition didactique: du savoir savant au savoir enseigné. Paris, Francia: La Pensée Sauvage.

Chevallard, Y. (1999). El análisis de las prácticas docentes en la teoría antropológica de lo didáctico. , 19(2), 221-266.

Chevallard, Y. (2004). Vers une didactique de la codisciplinarité. Notes sur une nouvelle épistémologie scolaire. Journées de didactique comparée, Lyon.

Chevallard, Y. (2007). Passé et présent de la théorie anthropologique du didactique. Sociedad, escuela y matemáticas. Aportaciones de la Teoría Antropológica de lo Didáctico, 705-746.

Cordero, F. (2003). Reconstrucción de significados del Cálculo Integral. La noción de acumulación como una argumentación. México: Grupo Editorial Iberoamericana.

Costa, V. A. \& Rossignoli, R. (2017). Enseñanza del algebra lineal en una facultad de ingeniería: Aspectos metodológicos y didácticos. Revista Educación en Ingeniería, 12(23), 49-55.

Costa, V. A. \& Vacchino, M. C. (2007). La enseñanza y aprendizaje del Álgebra lineal en la Facultad de Ingeniería, UNLP. En Congreso Chileno de Educación en Ingeniería, Chile.

Costa, V., Rossignoli, R., Sorichetti, C. \& Vampa, V. (2018). Algebra Lineal con Aplicaciones Parte I. Universidad Nacional de La Plata, La Plata: EDULP.

Costa, V.A. \& Justo, C.E. (2015). El álgebra lineal en la resolución de problemas altimétricos de topografía. En Encuentro Educación Matemática en Carreras de Ingeniería, Argentina.

Cumbal, L. C. (2012). La modelación matemática como proceso de estudio en el álgebra escolar. En 13 Encuentro Colombiano de Matemática Educativa, ECME-13 (ASOCOLME)

Decreto 792 de 2001. Diario Oficial No. 44.418 Ministerio de Educación Nacional, Bogotá, Colombia, 11 de mayo de 2001.

Farías, P. (2015). Diseño e implementación de una Actividad de Estudio e Investigación a partir de 
la pregunta ¿Cómo se puede medir un fractal teórico? (Tesis de Licenciatura). Universidad Nacional del Centro, Provincia de Buenos Aires Gómez-Chacón, I., \& Maestre, N. (2008). Matemáticas y Modelización. Ejemplificación para la enseñanza obligatoria. Experiencias de aula y experiencias didácticas, 17(1), 107-121.

Guerrero-Ortiz, C., \& Mena-Lorca, J. (2015). Modelación en la enseñanza de las matemáticas: Matemáticos y profesores de matemáticas, sus estrategias. Revista Electrónica de Investigación en Educación en Ciencias REIEC, 10(1), 1-13.

Hernández, R., Fernández, C., \& Baptista, M. (2014). Metodología de la Investigación. México: McGraw-Hill

Lay, D. (2007). Álgebra lineal y sus aplicaciones. México: Pearson Educación.

Liern, V. (2018). Algunos usos del Álgebra Lineal en las decisiones de Economía y Empresa. Universidad de Valencia, España.

Luzardo, D., \& Pena, A. J. (2006). Historia del Algebra Lineal hasta los Albores del Siglo XX. Divulgaciones Matemáticas, 14(2), 153-170.

Rojas, Á. \& Cano, A. (2009). Aplicaciones del Álgebra Lineal en la vida cotidiana. En XIV (Jornadas para el Aprendizaje y la Enseñanza de las Matemáticas. Girona, España

Mola, C., Montes, N., Rodríguez, M. L., Yordi, I. \& Sampedro, R. (2013). Estudio sobre la comprensióndelÁlgebra Lineal en los estudiantes de Ciencias Técnicas en la Universidad de Camagüey. Pedagogía Universitaria, 17(5), 2747.

Oktaç, A., \& Trigueros, M. (2010). ¿Cómo se aprenden los conceptos de álgebra lineal? RELIME: Revista latinoamericana de investigación en matemática educativa, 13(4), 373-385.

Otero, M. R., Fanaro, M., Corica, A., Llanos, V. C., Sureda, P., \& Parra, V. (2013). La Teoría Antropológica de lo Didáctico en el aula de Matemática. Buenos Aires: Editorial Dunken.

Otero, M., Fanaro, M., \& Llanos, V. (2013). La Pedagogía de la Investigación y del
Cuestionamiento del Mundo y el Inquiry: un análisis desde la enseñanza de la Matemática y la Física. Revista Electrónica de Investigación en educación en Ciencias, 8(1), 77-89.

Ottesen, J. (2001). Do not ask what mathematics can do for modeling. En D. Holton, \& (Ed), The teaching and learning of mathematics at the university level. An ICMI study (págs. 335 346). Dordrecht, The Netherlands: Kluwe.

Parraguez, M. (2013). El rol del cuerpo en la construcción del concepto Espacio Vectorial. Educación matemática, 25(1), 133-154.

Pochulu, M. (2018). La modelización en Matemática : marco de referencia y aplicaciones (1 ra. ed.). Villa María: GIDED.

Quiroz, S., \& Rodríguez , R. (2015). Análisis de praxeologías de modelación matemática en libros de texto de educación primaria. Educación matemática, 27(3), 45-79.

Rendón, M. (2016). Articulación entre la matemática y el campo de acción de la ingeniería de diseño de producto: aportes de la modelación matemática. Tesis doctorado. Medellín: Universidad de Antioqua.

Roa-Fuentes, S. \& Oktaç, A. (2010). Construcción de una descomposición genética: análisis teórico del concepto transformación lineal. Revista latinoamericana de investigación en matemática educativa, 13(1), 89-112.

Rodríguez, R. (2010). Aprendizaje y enseñanza de la modelación: el caso de las ecuaciones diferenciales. Revista Latinoamericana de Investigación en Matemática Educativa RELIME, 13(4-1), 191- 210.

Ruiz, A. (2001). Asuntos de método en la Educación Matemática. Revista Digital: Matemática, Educación e Internet, 2(1).

Salett, M., \& Hein, N. (2004). Modelación matemática y los desafíos para enseñar matemática. Educación Matemática, 16(2), 105 125.

Salgado, H. (2015). El papel de la modelación en la enseñanza de conceptos abstractos del álgebra lineal. Tesis doctorado. México: Instituto 
Politécnico Nacional.

Salgado, H. \& Trigueros, M. (2014). Una experiencia de enseñanza de los valores, vectores y espacios propios basada en la teoría APOE. Educación matemática, 26(3), 75-107.

Scardigli, M., Bello, C., Cicchini, A., Cuadrado, G. y Sara, A. (2013). Reflexiones sobre la Modelización Matemática como una Práctica de Enseñanza y de Aprendizaje en Carreras de Ingeniería. Revista de Informática Educativa y Medios Audiovisuales. 10(17), 17-21.

Siero, L. (2017). Diseño de una secuencia didáctica de modelación matemática para la enseñanza del álgebra lineal en la formación de ingenieros. Tesis doctoral. México: Instituto Politénico NAcional.

Suárez, L. (2014). Modelación-Graficación para la matemática escolar. México: Ediciones Díaz de Santos.

Suárez, L., \& Cordero, F. (2010). Modelación Graficación, una categoría para la matemática escolar. Resultados de un estudio socioepistemológico. Revista latinoamericana de investigación en matemática educativa RELIME, 13(4-11), 319-333.

Tenorio, Á. F., Martin, A. M. y Paralera, C. (2011). Introducción del Álgebra Lineal en la Economía: Una aproximación histórica. En Anales de ASEPUMA, (19), 0101, (pp. 1-20).

Trigueros, M. (2009). El uso de la modelación en la enseñanza de las matemáticas. Innovación educativa, 9(46), 75-87.

Trigueros, M., Maturana, I., Parraguez, M. y Rodríguez, M. (2015). Construcciones y mecanismos mentales para el aprendizaje del teorema matriz asociada a una transformación lineal. Educación matemática, 27(2), 95-124.

Uzuriaga , V., \& Martínez , A. (2016). Un ejemplo de evaluación desde un enfoque desarrollador, caso Álgebra Lineal. Scientia et technica, 21(3), 264-268.

Vasco, C. (2006). El pensamiento variacional, la modelación y las nuevas tecnologías. En C. Vasco, Didáctica de las matemáticas: artículos selectos (págs. 134-148). Bogotá: Universidad Pedagógica Nacional.

Vázquez, R., Romo, A., Romo-Vázquez, R., \& Trigueros, M. (2016). La separación ciega de fuentes: un puente entre el álgebra lineal y el análisis de señales. Educación matemática, 28(2), 31-57

Villa, J. A. (2007). La modelación como proceso en el aula de matemáticas. Un marco de referencia y un ejemplo. TecnoLógicas, 19, 63-85.

WunaJeeh, D., Osama, S., \& Juhaina, S. (2015). Discursive positionings and emotions in a small group's learning of geometric definitions. Ninth Congress of the European Society for Research in Mathematics Education CERME (págs. 11601166). HAL Archives-Ouvertes. 